\title{
Mitochondrial oncobioenergetics of prostate tumorigenesis (Review)
}

\author{
PRAVEEN KUMAR VAYALIL \\ Department of Medicine, University of Alabama at Birmingham, Birmingham, AL 35294, USA
}

Received March 4, 2019; Accepted July 2, 2019

DOI: $10.3892 / \mathrm{ol} .2019 .10785$

\begin{abstract}
Mitochondria are emerging as key players in the tumorigenic process of cells by maintaining the biosynthetic and energetic capabilities of cancer cells. It is now evident that mitochondria are involved in the malignant transformation, cell proliferation, aggression and metastatic behavior of prostate cancer (PC). Recent comprehensive analysis of the mitochondrial oncobioenergetic (MOB) profile of PC cells using microplate-based high resolution respirometry has clearly demonstrated that characteristic MOB alterations occur at different stages of PC development. Additionally, studies have reported that modification of the MOB profile significantly inhibits the growth of malignant cells. This observation suggests that dynamic alterations in the MOB function are a critical component in the development of malignant disease of the prostate. Therefore, quantification of MOB function may be a good marker for the prediction of tumor stage. Future studies may develop novel approaches to measure oncobioenergetics of tumors with minimal invasive procedures effectively in men to determine the general prostate health and tumor staging, and to predict whether a tumor will proceed to malignancy in patients. Additionally, since $\mathrm{PC}$ is a slow growing tumor, modulating the MOB profile at specific stages of tumor development may be a novel approach to treat or prevent PC.
\end{abstract}

\section{Contents}

1. Introduction

2. Intermediary metabolism of prostate peripheral zone epithelium

Correspondence to: Dr Praveen Kumar Vayalil, Department of Medicine, University of Alabama at Birmingham, 901 19th Street South, Birmingham, AL 35294, USA

E-mail: praveen@uabmc.edu

Key words: mitochondria, bioenergetics, oncobioenergetics, mitochondrial oncobioenergetic index, prostate cancer, fatty acid oxidation, oxidative phosphorylation, glycolysis, intermediary metabolism, net citrate synthesis
3. Defining mitochondrial oncobioenergetic profile and mitochondrial oncobioenergetic index

4. Oncobioenergetic changes during prostate carcinogenesis

5. Mitochondrial oncobioenergetic index of prostate tumorigenesis

6. Limitations of XF technology

7. Conclusion

\section{Introduction}

Prostate cancer (PC) is a disease of the old age (1). The specific underlying mechanisms of prostate carcinogenesis have not been unraveled yet. The only well-established risk factors for PC are older age, black race/ethnicity, and a family history of the disease $(2,3)$. Therefore, future progress in combating PC will be highly dependent upon an understanding of the mechanisms involved in the development and steady progression into prostate malignancy.

Mitochondria are emerging as key players in the tumorigenic process of cells by maintaining the biosynthetic and energetic capabilities of cancer cells. Besides compartmentalizing different metabolic pathways, the mitochondria is engaged in the generation of much of the cellular energy, regulation of the redox state of the cell, generation of reactive oxygen species (ROS), buffering $\mathrm{Ca}^{2+}$ and initiating apoptosis (4). Mitochondria are involved in the final stage of the cellular catabolism and maintain the redox homeostasis at different levels. Through several enzymatic reactions, carbohydrates, fats and proteins are degraded into smaller molecules, which is further converted to pyruvate by glycolysis, fatty acids and amino acids (Fig. 1). Mitochondria further transform these small molecules into $\mathrm{NADH}$ and $\mathrm{FADH}_{2}$ (reduced energy equivalents), through $\beta$-oxidation and TCA cycle or rerouted to biosynthetic pathways. The reduced energy equivalents are then utilized by the mitochondrial electron transport chain (ETC) through oxidative phosphorylation (OXPHOS). The electrons liberated by the oxidation of NADH and $\mathrm{FADH}_{2}$ are passed along a series of carriers of ETC located in mitochondrial inner membrane. The electrons are ultimately transferred to molecular oxygen to form water (Fig. 2). ETC consists of four enzyme complexes (complexes I-IV), and two electron carriers (coenzyme Q and cytochrome c). These complexes are composed of numerous protein subunits encoded by nuclear and mitochondrial genes, except complex II, which are encoded by nuclear genes only. 
In addition, the electron-flavoprotein (ETF) system, which involves $\mathrm{ETF}_{\mathrm{A} / \mathrm{B}}$ and the ETF-QO proteins, connects fatty-acid oxidation with coenzyme $Q$ reduction. The glycerol-3-phosphate dehydrogenase also participates in redox homeostasis by oxidizing cytosolic NADH to reduce mitochondrial FAD to $\mathrm{FADH}_{2}$. Finally, the malate-aspartate shuttle (NADH-shuttling) also fuels OXPHOS and supports redox homeostasis via the delivery of cytosolic NADH to the mitochondrial matrix. The oxidation of NADH or $\mathrm{FADH}_{2}$ by complex I or complex II, respectively, triggers the transfer of electrons from complex I (or II) to complex IV. Meanwhile, protons are pumped from the matrix into the inter-membrane space, thus generating an electrochemical gradient of protons which is finally used by the $\mathrm{F}_{1} \mathrm{~F}_{0} \mathrm{ATP}$ synthase (i.e., complex V) to produce adenosine triphosphate (ATP), the main energetic currency of the cell (5).

Mitochondrial function, its genetics and energy metabolism have important roles in tumor initiation and progression and are considered emerging indicators of PC biology (6). There is increasing evidence that key oncogenes and tumor suppressors modulate mitochondrial dynamics through important signaling pathways. Thus, mitochondrial mass and function vary between tumors and individuals, but, the significance of these events for cancer is not fully appreciated (7). Therefore, in the present review, we emphasize on the current knowledge on the mitochondrial bioenergetics of normal prostate epithelial cells (PECs; normal physiology) and as they transform from indolent tumor to aggressive PC (pathology).

\section{Intermediary metabolism of prostate peripheral zone epithelium}

The major characteristic of PECs of the peripheral zone of the prostate is that they accumulate four times higher concentrations of citrate compared to epithelial cells from other mammalian tissues. PECs secrete citrate into the prostatic fluid at a concentration of 3-10 times higher than the prostate tissue levels itself (8). This metabolic transformation of PECs during cellular differentiation is referred to as 'net citrate production' (8). In parallel, the prostate peripheral zone accumulates extremely high levels of zinc in the range of 10-15 times higher compared to other tissues $(8,9)$. Current hypothesis, based on the evidence, is that increased accumulation of citrate is due to the inhibition of aconitase by high levels of zinc, which inhibits the conversion of citrate to aconitate. This results in truncated Krebs cycle and reduced OXPHOS (10). Consequently, to meet the cellular energy demands and synthesize large amounts of citrate, precursor requirements of PECs are different and exceed from that of other normal cells.

In the citrate producing PECs, instead of being oxidized, citrate becomes an end-product of TCA cycle (9). To maintain high levels of citrate, PECs require substantial amounts of acetyl CoA and oxaloacetate for its synthesis by citrate synthase. Therefore, under these metabolic demands, availability of precursors for citrate synthesis is crucial. Recent evidence indicates that PECs have highly flexible metabolism $(11,12)$. PECs could meet the high precursor requirements through the utilization of five different substrates such as glucose, pyruvate, aspartate, glutamine, and fatty acids. Cells can utilize these substrates based on its availability. However, glucose seems to be the preferred substrate. Studies have shown that PECs consume high amounts of glucose (13-15) and have higher aerobic glycolysis (10) compared to other cell types $(11,16)$, such as normal breast epithelial cells or cells with rapid cell turnover such as monocytes (17). Acetyl CoA is mainly generated from pyruvate and $\beta$-oxidation of fatty acids. Although, pyruvate is the end-product of glycolysis and is the major source of pyruvate, it can also be generated from other sources and could be utilized for the synthesis of acetyl CoA. We have recently shown that RWPE-1 cells could efficiently oxidize both endogenous as well as exogenous fatty acids significantly (11). We have also shown that glutamine could be utilized both as a substrate for energy and citrate production by gaining access into the TCA cycle by its conversion to $\alpha$-ketoglutarate through the enzymatic action of glutaminases followed by glutamate dehydrogenase (11). Consequently, glutamine could be converted to OAA for citrate synthesis and at the same time generate 9 ATP's in PECs with a truncated TCA cycle compared to 12 ATPs made when an acetyl CoA molecule completes one TCA cycle (Fig. 1). Aspartate could be converted by PEC's to produce OAA (8-10). It is catalyzed by glutamate oxaloacetate transaminase (GOT). GOT catalyzes the interconversion of aspartate and $\alpha$-ketoglutarate to oxaloacetate and glutamate (Fig. 1). High aspartate levels were maintained by transporting it into the cell from blood by taking advantage of a $\mathrm{Na}^{+}$-coupled co-transporter EAAC1 (18). Another anaplerotic pathway that may be important in providing OAA for citrate synthesis is the conversion of pyruvate to OAA catalyzed by pyruvate carboxylate $(\mathrm{PyC})$. This pathway may be more efficient in generating OAA as the cells are highly glycolytic producing large amounts of pyruvate and part of it could be shunted for OAA synthesis. However, the importance of PyC in PECs is still elusive. Overall, it should be concluded that metabolic plasticity is an inherent feature of citrate producing PECs and these cells may acquire these characteristics as they functionally differentiate.

\section{Defining mitochondrial oncobioenergetic profile and mitochondrial oncobioenergetic index}

Metabolic profile of a cell is governed by the way which the mitochondria functions in a cell. Any change in mitochondrial function would alter the cellular metabolic profile. Thus, under physiological conditions, the term mitochondrial bioenergetics indicates how mitochondria functions or its functional status/profile that allows the cells to meet the metabolic demands. Several studies have already been conducted to report the metabolic profile of cancer cells. However, how the mitochondria functions or what is the mitochondrial bioenergetics during the evolution of cancer that support its metabolic requirements are still unexplored.

MOB may be simply stated as the mitochondrial bioenergetics that is similar to that of a cancer cell. It is defined as stable (at least in cell and ex vivo models), measurable, change in the mitochondrial bioenergetics during the course of cell transformation from normal to malignant tumor (11). It indicates the mitochondrial bioenergetics or the functional state of the mitochondria that predisposes, promotes or favors carcinogenesis. It is dynamic in nature (11) and depends on the stage 


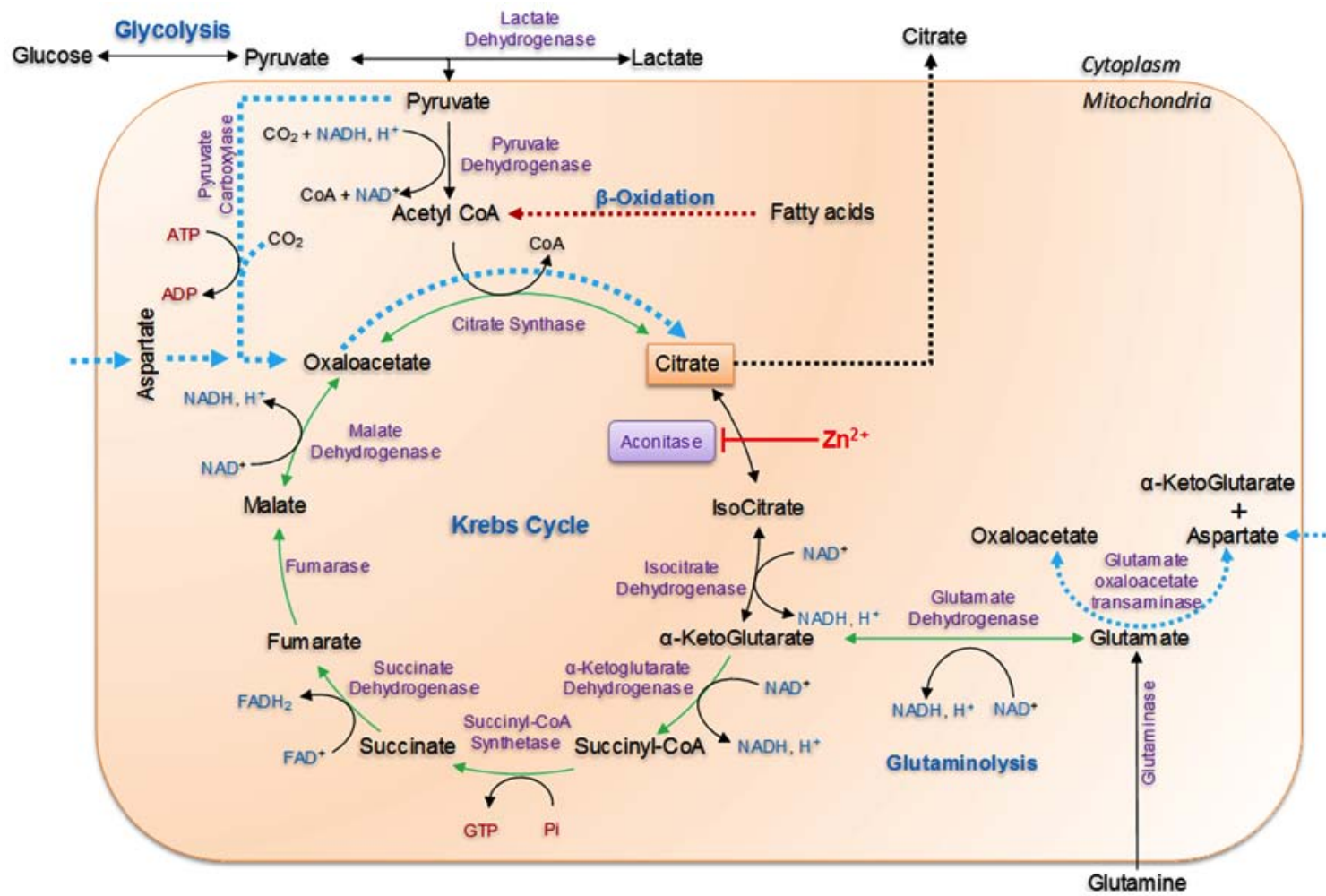

Figure 1. Intermediary metabolism in prostate epithelial cells. The major substrates and signaling pathways utilized in the truncated TCA cycle for citrate accumulation are depicted. The major substrates that are fed into the Krebs cycle are pyruvate via glucose, which may be converted to acetyl coA by pyruvate dehydrogenase and OAA by pyruvate decarboxylase. Glutamine is fed into the TCA cycle as $\alpha$-ketoglutarate by the action of glutaminase and glutamate dehydrogenase. Aspartate is directly taken into the cell and transaminated by glutamate oxaloacetate transaminase into OAA. Purple represents enzymes, cofactors are presented in blue, glutaminolysis in green, nucleotides in red and substrates in black. OAA, oxaloacetate; TCA, tricarboxylic acid.

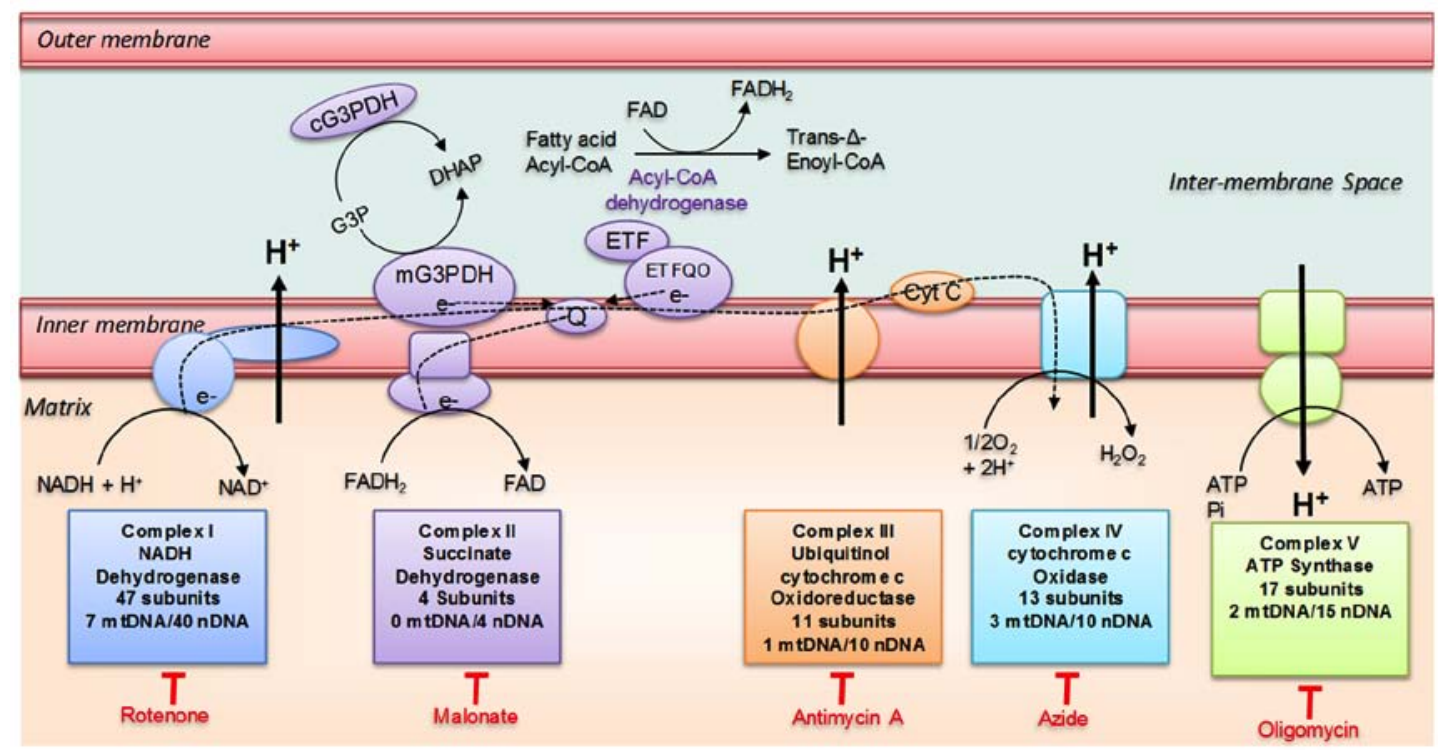

Figure 2. Diagrammatic representation of the mammalian ETC. Mitochondrial respiratory chain consists of four membrane-bound enzyme complexes (I-IV) and three of these are proton pumps. In addition, the ETC also contains two intermediary substrates (coenzyme Q and cytochrome c). The $\mathrm{NADH}^{+} \mathrm{H}^{+}$and $\mathrm{FADH}_{2}$ produced by the intermediate metabolism are oxidized further by the mitochondrial respiratory chain to establish an electrochemical gradient of protons, which is finally used by the F1F0-ATP synthase (complex V) to produce ATP, which is the only form of energy used by the cell. Electrons can also be delivered to the respiratory chain at the level of coenzyme Q by the ETF system or by the G3PDH system. ETC, electron transport chain; ETF, electron-transferring flavoprotein; G3PDH, glycerol 3 phosphate dehydrogenase; mtDNA, mitochondrial DNA; nDNA, nuclear DNA.

of the tumor $(11,16)$, tissue origin and microenvironment (19). It may be mathematically represented as mitochondrial oncobioenergetic index (MOBI) (11) (see below). MOBI is not only associated with cancer cells but also with the individual cellular components of the tumor, such as fibroblasts, immune cells etc., that support cancer cell growth at each stage of the tumor 
development. It also represents mitochondrial bioenergetics of cancer tissues itself and the normal tissues that are highly vulnerable to carcinogenesis. MOB of a tissue represents or is the result of both the metabolic rewiring of the cancer and other tumor associated cells induced during transformation and pathological stress induced by tumor growth.

It is now evident that mitochondria are involved in malignant transformation, cell proliferation, aggression, and metastatic behavior of prostate cancer $(6,20)$. Recent comprehensive analysis of the oncobioenergetic profile of PC cells using microplate-based high resolution respirometry has clearly demonstrated that characteristic MOB changes occurs at different stages of PC development (11). Modification of MOB significantly inhibits the growth of malignant cells $(21,22)$. This observation suggests that dynamic changes in the MOB function are a critical component in the development of malignant disease of the prostate.

Different parameters of mitochondrial oncobioenergetic (MOB) function could be established by using microplate (23) or Oxygraph high resolution respirometry (24). Microplate assay provides a high-throughput approach, which provide valuable information on the various aspects of mitochondrial function through mitochondrial stress test (MiST) $(25,26)$. The function of mitochondria is directly related to the amount of oxygen consumed and various parameters are derived from the oxygen consumption rate (OCR) trace after interrogation with compounds that can modulate the mitochondrial function (Mito-effectors; Fig. 3). Major functional parameters of a MiST are the following:

Basal oxygen consumption. It represents the amount of oxygen consumed by the mitochondria, which allows the cells to support the fundamental cellular functions under optimal physiological conditions. Basal oxygen consumption contributes to the synthesis of ATP at complex V and proton leak (see below). A change in basal rate in each cell type indicates some bioenergetic change in the cell, which can be estimated by performing MiST. Basal respiration can alter dramatically, due to inherent properties of the cells, such as metabolic rewiring, as described above. It can also be affected by the presence or absence of different substrates (glucose, pyruvate, lactate, fatty acids, glutamine, oxygen), growth factors and hormones, $\mathrm{pH}$ and osmolality in the environment (26). Therefore, optimal concentrations of these substrates should be employed for analysis of the mitochondrial function. For example, glucose concentrations above $10 \mathrm{mM}$ may significantly inhibit the basal respiration of different cell types. The mean normal blood glucose level in humans is about $5.5 \mathrm{mmol} / 1(100 \mathrm{mg} / \mathrm{dl})$. Therefore, it is always preferable to use physiological concentration of glucose for cellular respiration assays. Moreover, the availability of different substrates in the surrounding environment may also influence the basal oxygen consumption.

ATP-dependent oxygen consumption. Otherwise described as oligomycin sensitive oxygen consumption, ATP-dependent oxygen consumption represents the amount of oxygen consumed to generate ATP at complex V or ATP synthase. It is determined by measuring the OCR after interrogating with oligomycin. It is the difference between basal oxygen

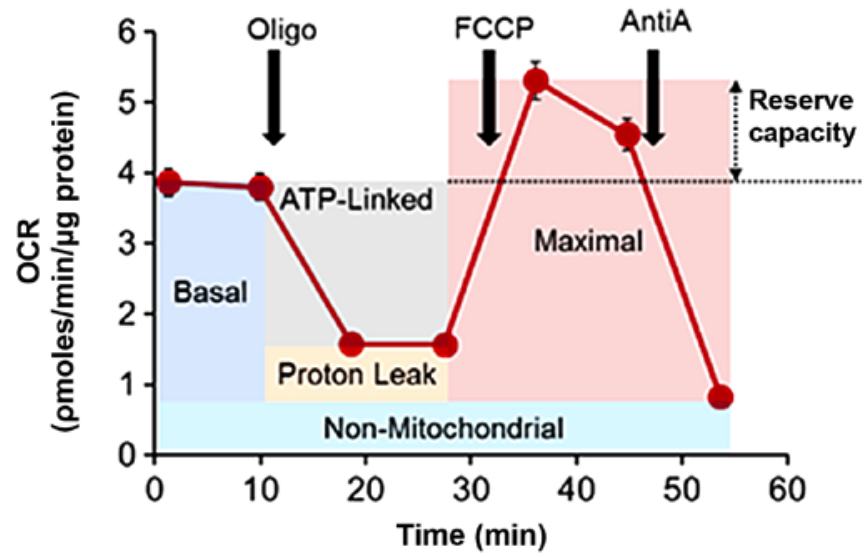

Figure 3. Measurement of mitochondrial bioenergetic function using the XF24 analyzer and determination of different mitochondrial bioenergetic parameters from OCR profile. Seahorse Bioscience V7 Tissue Culture Plates were seeded with optimal cell density and allowed to grow for $24 \mathrm{~h}$ prior to the measurement of OCR. Basal OCR was measured three times followed by the sequential addition of Oligo, FCCP and AntiA with a measurement of OCR. Colored boxes in the OCR trace represent the relative contribution of basal oxygen consumption, non-respiratory chain oxygen consumption, ATP-linked oxygen consumption, the maximal OCR after the addition of FCCP, proton leak and the reserve capacity of the cells. AntiA, antimycin A; FCCP, carbonyl cyanide-p-trifluoromethoxyphenylhydrazone; OCR, oxygen consumption rate; Oligo, oligomycin.

consumption and the oxygen consumption due to oligomycin inhibition of complex V (25). Inhibition of ATP synthase would increase the proton gradient (mitochondrial hyperpolarization), which in turn prevents the electron flow through other complexes. This inhibition results in reduced oxygen consumption. Therefore, any increase in ATP-dependent OCR implies a higher ATP demand. On the other hand, a decrease in ATP-dependent OCR represents a reduced ATP demand, a lack of substrate availability and/or severe damage to OXPHOS (25).

Proton leak. Proton leak is a physiologic process, which represents reentry of protons into the mitochondrial matrix through facilitated diffusion without contributing to ATP synthesis and energy dissipated as heat. Proton leak can be an intrinsic property of the cell or induced. The intrinsic proton leak is unregulated, cell-type specific and correlates with metabolic rate of the cell. Intrinsic proton leak is mainly mediated by mitochondrial anion carriers and is minimal through the lipid bilayer. Inducible leak occurs through the adenine nucleotide translocase (ANT) and uncoupling proteins (UCPs) and can be initiated by fatty acids, superoxide, or peroxidation products (27). The physiological role of inducible leak through UCP1 in mammalian brown adipose tissue is heat production (27).

An increase in intrinsic leak could be attributed to damage of the inner mitochondrial membrane or ETC complexes. This disproportionate increase in oxygen consumption is termed 'electron slip,' where electrons are transferred through the ETC complexes (Complexes I, III and IV) without pumping protons into the intermembrane space. There is also evidence to show that oxidative stress capable of damaging the mitochondrial membrane or ETC complexes could increase ATP-dependent oxygen consumption and proton leak (28). 
Maximum oxygen consumption. Use of uncouplers (e.g. FCCP or carbonyl cyanide trifluoromethoxy phenylhydrazone) to generate artificial energy demand would allow measuring the maximum possible mitochondrial oxygen consumption under the experimental conditions where substrate concentration is not limiting. Uncouplers disrupt the electrochemical gradient across the membrane and inhibit the coupling between the electron transport and phosphorylation reactions. Thus, by uncoupling, electron flow and ATP synthase activity are maintained and at the same time the oxygen consumption is elevated without ATP synthesis. During artificially induced uncoupling, glucose/pyruvate/glutamine/fatty acids are continuously utilized to generate reducing equivalents (NADH and $\mathrm{FADH}_{2}$ ) to run the ETC at its maximum rate as an attempt to restore the electrochemical gradient across the membrane. A high maximal respiration compared with basal OCR implies that the cells require or uses only partial capability of the mitochondria to support the energy demand even in the presence of ample amounts of substrate.

A reduction in maximum oxygen consumption is a strong indicator of potential mitochondrial dysfunction or metabolic rewiring as in the case of cancer cells. A low maximal capacity could also indicate decreased activity of metabolic pathways that produce reducing equivalents, substrate availability, mitochondrial mass or integrity is compromised.

Concept of reserve capacity. The spare or reserve capacity is a well-established concept $(25,29,30)$. This value denotes the difference between the maximal and the basal respiration. It represents the ability of the mitochondria to respond to an increase in energy demand. Conceptually, reserve capacity indicates how close to its bioenergetic limit a cell is operating. Thus, the reserve or spare capacity describes an estimate of the potential bioenergetic reserve the cell can call upon in times of stress $(25,29,30)$. It has been shown that cancer cells under conditions of stress, such as targeted thiol modification of mitochondrial proteins that affect the mitochondrial protein function, the reserve capacity is completely depleted and if the threshold for the basal respiration is breached then cell death occurs $(16,21,22)$. Whether cells can utilize the maximal electron transport activity for ATP synthesis will depend on the capacity of the components of the ETC and oxidative phosphorylation system (25). From a translational point of view, alterations in reserve capacity of tumor cells may be linked to their changing biology during the progression of the disease (11).

$M O B I$. Mitochondrial dysfunction, for example due to stress, is characterized by a loss of efficiency in the electron transport chain and reductions in the synthesis of high-energy molecules, such as ATP (31). Alternative to mitochondrial dysfunction, we introduce MOBI, which denotes a mathematical representation or quantitative assessment of the oncobioenergetics due to cell transformation. It indicates a stable change in mitochondrial bioenergetics because of metabolic rewiring that occurs at various stages of oncogenesis to meet different cellular functional requirements, such as proliferation, invasion and metastasis. It may represent a higher, or lower cellular mitochondrial function compared to the normal cell population. MOBI is derived from the parameters of mitochondrial stress test (MiST) and is calculated from the following formula modified from previously described (11).

MOBI = Basal Mito. Resp. $\times \log ($ Net Mitochondrial Efficiency $) \quad E q-1$

Where, the net mitochondrial efficiency $(E q-2)$ is a constant for each cell type at a defined optimal set of conditions (data unpublished). Under these conditions, it is possible to compare the MOBI of a normal cell with another transformed phenotype. Thus, higher net mitochondrial efficiency indicates functionally efficient mitochondria and vice versa.

The net mitochondrial efficiency is defined as

Net Mitochondrial Efficiency $=\frac{(\text { ATP Dep. Resp. }+ \text { Reserve Cap. })}{\text { Proton Leak }} \quad$ Eq-2

Where, basal mitochondrial respiration (Basal Mito. Resp.), ATP dependent respiration (ATP Dep. Resp.), and reserve capacity (Reserve Cap.) were used in the equations. MOBI was found to be a valuable tool to distinguish the aggressiveness of different PC cells of different stages (11) and possibly in other cancers too. However, further studies are required to establish this notion.

\section{Oncobioenergetic changes during prostate carcinogenesis}

Mitochondrial bioenergetic profile of normal peripheral zone PECs. Basal mitochondrial function and ATP requirements of PECs could be achieved efficiently by utilizing glucose, glutamine or fatty acids as their energy substrate depending on their bioavailability (11). This is comparable with that of other cells such as breast epithelial cells or cells with rapid turnover, such as monocytes. In presence of glutamine alone, the basal respiration increases sharply to meet the energy demands, because ATP generation through glycolysis is significantly low or absent.

One of the major mitochondrial bioenergetic functions of a cell is its reserve capacity. PECs demonstrate a variable reserve capacity depending on the substrate utilization. In presence of glucose, PECs have a very low reserve capacity. However, in presence of glutamine, the cells have a very high reserve capacity. This may be due to truncated Krebs cycle, where in presence of glucose, much of the ATP generation is met through glycolysis. Glutamine on the other hand fed into the Krebs cycle as $\alpha$-ketoglutarate, which simultaneously generates ATP and produce metabolites for the synthesis of citrate such as OAA (Fig. 1). It seems that cells prefer glucose over glutamine as combined use of both substrates did not elevate reserve capacity like that of glutamine alone. However, further studies are essential to establish substrate specificity of PECs.

Other notable feature of PECs is the bioenergetic phenotype which is distinct from other cell types. Majority of the normal epithelial cells have either a resting or aerobic phenotype $(11,16,21)$. PECs are specialized cells and are highly energetic that consume large amount of energy producing substrates, especially glucose, to maintain the metabolic needs of the cells and is necessary as they accumulate and secrete enormous amounts of citrate.

Utilization of fatty acids to maintain the mitochondrial respiration of PECs is poorly understood. Recent studies show that PECs could significantly mobilize endogenous fatty acids 
and can utilize both endogenous and exogenous fatty acids to maintain the mitochondrial respiration (11). Our studies show that endogenous as well as exogenous fatty acids could elevate reserve capacity significantly even with a truncated TCA cycle through the generation of NADH and FADH2 during $\beta$-oxidation and possibly through glycerol-phosphate shuttle (Fig. 2). This reinforces the idea that PECs can utilize all energy producing substrates efficiently to maintain its cellular metabolic function or requirements that are unique to PECs.

Glycolytic profile of prostate tumorigenesis. PECs are generally glycolytic. Glycolysis does not change drastically at early non-malignant stages compared to PECs and maintain the basal rate but changes as they become malignant. As the cells become malignant, the glycolytic capacity (peak glycolytic activity), and glycolytic reserve (available glycolytic activity at any time when stressed) are all elevated (11). Deriving the cellular Oncoglycolytic Index (OGI) from GlyST would be very useful to define the metabolic rewiring during the transformation of PECs from indolent to aggressive prostate cancer. Combining OGI and MOBI would be useful parameters in defining the oncogenicity of the tumor. Our ongoing studies are currently in this direction.

Factors affecting mitochondrial function during prostate tumorigenesis. Prostate tumor growth and development into a malignant form occurs over several years. During this period, tumor undergoes various changes at tissue, cellular, genetic and biochemical levels. Due to reduced tumor vasculature and increase in size of the tumor, both PC cells as well as stromal cells must 'functionally adapt' to the microenvironment within the tumor mass and mutually support their growth in a hostile environment (32). There are factors that permanently or transiently alter the mitochondrial bioenergetics of PECs as they progress from indolent to invasive and metastatic cancer. In addition to nuclear and mitochondrial genetic mutations, the major environmental factor that molds the biology of cancer cells and stromal components is low $\mathrm{O}_{2}$ tension as well as availability of nutrients within the tumor. The key cellular organelle that is functionally affected by changes in $\mathrm{O}_{2}$ tensions and supply of energy substrates is the mitochondria. Therefore, modulation of mitochondrial function or oncobioenergetics of tumor at each stage of the tumor development is crucial for the successful growth, progression and dissemination of the tumor.

The most common known genomic alterations in $\mathrm{PCa}$ involve four pathways/genes: the androgen receptor pathway, PI3K pathway, rearrangements that place members of the ETS transcription factor family under control of androgen responsive promoter TMPRSS2, and loss of function of the prostate tumor suppressor NKX3.1 (33). These pathways can contribute directly or indirectly to changes in mitochondrial function. For example, PI3K/AKT activation leads to increased mitochondrial respiration by increasing substrate supply to mitochondria, enhancing the mitochondrial catalytic machinery such as pyruvate dehydrogenase activity and up-regulation of mitochondrial electron transfer and energy-transduction capacity through complex I and phosphorylating ATP synthase (34).

The only human cellular cytoplasmic organelle that possess their own functional DNA are the mitochondria (35). Most mtDNA copies are identical at birth (homoplasmy).
However, mtDNA molecule has no adequate proofreading and repair mechanisms, as it is exposed to an environment with high concentrations of ROS. Thus, mtDNA molecule mutates at a rate that is about 10-100 times higher than nDNA. Occasionally, a subpopulation of mtDNA molecules carries a pathogenic mutation (heteroplasmy) and has been shown to promote tumorigenesis $(35,36)$. Many studies have shown that mtDNA homoplasmic and heteroplasmic mutations in PC are very common, perhaps due to increased ROS production (37-52) and have been reviewed comprehensively elsewhere $(6,46)$. In an extensive well-controlled mitochondrial DNA sequence analysis (6), it was concluded that in addition to frequent somatic mtDNA mutations in PC cells, normal appearing benign cells surrounding the tumor harbor somatic mtDNA mutations (field cancerization effect) as well, (c) these mutations demonstrate a progressive pattern of malignant disease and finally, (d) some of these mutations are linked to PCa development. Thus, these studies convey the idea that PCa development involves changes in the mitochondrial oncobioenergetics through mtDNA mutations and may allow the cells to meet the metabolic demands during transformation.

Another major factor that may rapidly influence the mitochondrial function is the tumor microenvironment. Microenvironment consists of insoluble extracellular matrix (proteins, glycoproteins, proteoglycans and polysaccharides), a stroma (non-cancerous cells such as fibroblasts, adipose immune cells and endothelial cells), and several growth factors and cytokines secreted by stromal components. Cancer cells are embedded in the tumor encapsulated by stromal cells. Microenvironment provides a metabolically friendly environment for cancer cells to grow and multiply within the tumor. They supply $\mathrm{O}_{2}$ and energy producing substrates (glucose, fatty acids and glutamine) required to meet high energy demands of cancer cells. As the tumor grows, their energy demands are so high, and stroma induces abnormal angiogenesis and nutrients are delivered through aberrant blood vessels. This generates hypoxia and nutrient deprivation within the tumor. It has been reported that many prostate cancers are hypoxic, and the distribution of oxygen values is similar to other human tumors (53). The major organelle that is affected by low oxygen and nutrient deprivation is the mitochondria and it must adapt to the ever-changing microenvironment for the survival of cancer cells under these severe conditions. Hypoxia can cause changes in mitochondrial structure and dynamics such as impairment of mitochondrial fusion, cause mitochondrial depolarization, and loss mitochondrial DNA within the cells. Hypoxia can cause changes in the activity of metabolic enzymes such as citrate synthase, respiratory complexes such as inhibition of cytochrome oxidases by nitric oxide generated through the activation of nitric oxide synthases, enhance super-complex disassembly and inhibition of complex $\mathrm{V}$ through ROS generation (54). Therefore, one of our hypotheses is that energy flow within the tumor tissue dynamically changes at each stage of the tumor development to support the tumor growth and progression into an aggressive tumor.

Mitochondrial oncobioenergetics of prostate epithelial cells upon transformation and at different stages of malignancy. To study the oncobioenergetic profile of PC cells at different 
Table I. Limitations of XF technology.

Item

Limitations

Injectable Cost of optimizing injectable concentrations and the subsequent cost per respiratory assay are high A maximum of four. It will be necessary to use multiple plates when more than four injectable reagents are required

Injectable compounds may potentially interfere with sensor fluorescence or the plastic plate and produce misleading data

Cell lines The generation of immortalized cell lines are limited due to loss of differentiated functions, high glycolytic rates, and reduced respiration

Since individual cell populations are analyzed, they lack direct comparisons with whole - tissue measurements that may contain multiple cell types.

Mitochondrial responses to substrates such as ADP, creatine, or cytochrome c cannot be investigated in intact preparations because they are unable to penetrate the cellular membrane.

Pharmaceutical Requires optimization for each cell line. This is to ensure that they do not interfere with other inhibitors and produce misleading data

Contamination Contamination by viruses, mycoplasma, and other cell lines

stages of cancer development, a model with genetically identical clones of cells is required that recapitulate the multi-stage carcinogenesis process. Recently, using RWEP1 and malignant clones derived from this cell line, we demonstrated that PC cells at each stage show characteristic oncobioenergetic features as they grow from an indolent tumor to an aggressive tumor. Our studies showed that immediately after transformation, the cells achieve significantly higher OXPHOS and decrease further as the cells achieve malignancy. This early switch in bioenergetics may be due to reduced zinc accumulation, restoration of aconitase activity, and functioning of TCA cycle without truncation (8-10). These changes are also reflected in various mitochondrial bioenergetic parameters such as basal, maximal, and ATP-dependent respiration as they are elevated during early non-malignant stages and decreases with increasing malignancy irrespective of the substrates used, such as glucose or glutamine. Although oncobioenergetics changes occur at each stage of tumor progression, the amount of ATP generated is comparable with that of the normal. On the other hand, both endogenous and exogenous fatty acid oxidation decreases with increasing malignancy suggesting that much of the fatty acids may be utilized for lipid synthesis for the biomass. All these data indicate that in prostate tumorigenesis, the mitochondrial bioenergetics of the tumor cells changes dynamically to functionally adapt to the stage of the tumor.

\section{Mitochondrial oncobioenergetic index of prostate tumorigenesis}

Different cellular bioenergetic parameters could be derived from MiST trace and these parameters are valuable to compare genetically identical cells at various stages of malignancy. However, tumor cells of varying degree of malignancy from genetically different cells have overlapping mitochondrial oncobioenergetic and metabolic phenotypes and each individual parameter does not correlate with the degree of malignancy and is less useful and has less clinical translational value. Recently we have shown that calculating

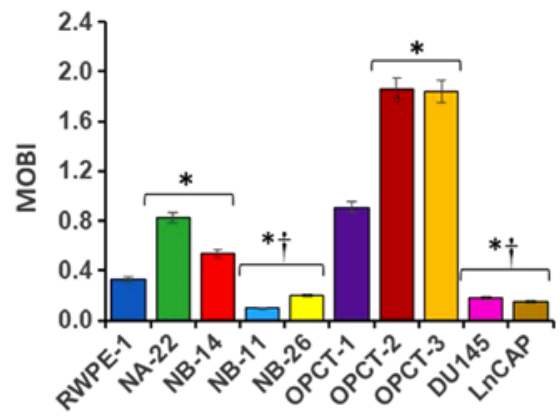

Figure 4. MOBI of PC cells obtained from RWPE-1 cells (NA-22-NB-26) and human tumors (OPCT-1-LnCAP cells). The data were analyzed using one-way ANOVA followed by Tukey's post hoc test. * $\mathrm{P}<0.05$ vs. RWPE-1 cells; ${ }^{\dagger} \mathrm{P}<0.05$ vs. OPCT-1 cells (11). MOBI, mitochondrial oncobioenergetic index. PC, prostate cancer.

MOBI of various PC cell lines of various malignant stages is very useful to differentiate one cell from one another based on their malignancy (Fig. 4). Our studies using ten PC cell lines demonstrated for the first time that each cell line has a unique MOBI, under standard assay conditions. It increases drastically as the prostate epithelial cells transform into localized pre-malignant stages of tumor compared to the normal PECs and it falls below the normal as soon as they become aggressive or malignant. MOBI seems to be independent of genetic similarity, androgen sensitivity or mitochondrial content determined recently for many PC cells (12), and is more related to their malignant stage. Although yet to define, MOBI specific for individual substrates and under stressed conditions or both would also provide valuable information that would allow defining the oncobioenergetics of cancer cells much more accurately at various stages of carcinogenic process. Therefore, these observations emphasize the importance of studying mitochondrial function of tumors within the context of the physiological environment to understand the mechanisms by which mitochondria contribute to the tumor pathology. 
MOBI could be a valuable tool for assessing the aggressiveness of the tumor as well as detect whether an indolent tumor may become aggressive in the future. It is also a valuable tool to test the metabolic health of prostate. Any abnormality in the mitochondrial bioenergetics of prostate tissue may represent a pathological condition. However, suitable techniques are not available to assess MOBI in the physiological environment without disrupting the interaction between the cell environment and the mitochondria in the tissues. Ex vivo approaches allow analysis of mitochondrial bioenergetics by rigorously controlling the assay conditions. Recent developments in technologies to measure mitochondrial bioenergetics have shown that tissue slices could be used for assessing the oncobioenergetics ex-vivo (55-57). Tissue slices essentially are viable explants of the tissue that can be cultured ex vivo and are widely used by many researchers for several years as a model of the organ under study including metabolism. Now digitally controlled tissue slicers are commercially available to prepare $200 \mu \mathrm{m}$ thick precision-cut tissue slices (58-60). Thus, it is possible to obtain tissue cores from specific locations and cut slices from specific depths within the tissues and could be compared with another slice obtained from same location and depth with minimum variations (58-60). However, some of the major issues confronted during the measurement of mitochondrial bioenergetics in tissue slices needs to be resolved. These include poor penetration of mito-effectors into the mitochondria and slow diffusion and depletion of substrates and oxygen during measurements. Hyperoxia and increasing substrate concentration is an option but may result in non-physiological response. Moreover, new algorithms should be developed by taking into consideration of all these impeding factors and corrected for accurate measurements.

Non-invasive techniques, such as imaging approaches for the evaluation of mitochondrial bioenergetics of cancer, are still in its infancy (61-64). It was mainly designed to characterize different aspects of mitochondrial dysfunction. It permits quantitative measurement of fluxes of ATP and oxygen, which are controlled by mitochondria. Thus, it allows determining the mitochondrial coupling efficiency $\left(\mathrm{ATP} / \mathrm{O}_{2}\right)$ as well as the tissue capacities for phosphorylation and oxidation. By these approaches, it has been shown to reveal important aspects of mitochondrial dysfunction with disease and age. However, the utility of these methods for quantifying the oncobioenergetics of tumors in vivo is yet to be discovered.

\section{Limitations of XF technology}

There are several limitations to consider when analyzing mitochondrial respiration using the Seahorse XF. They are given below (Table I).

\section{Conclusion}

Mitochondria play a significant role in prostate tumorigenesis and are modulated at each stage of the tumor development to meet the metabolic and/or bioenergetic requirements of the cell. Thus, quantification of mitochondrial oncobioenergetic function will be a good biomarker for predicting the tumor stage. Future studies would allow novel approaches to measure oncobioenergetics of tumor with minimal invasive procedures effectively in men to determine the general prostate health, tumor staging and predict whether a tumor will proceed to malignancy in patients. Moreover, as prostate is a slow growing tumor, modulating the mitochondrial bioenergetics at specific stages of tumor development will be a novel approach to treatment or prevention of prostate cancer.

\section{Acknowledgements}

Not applicable.

\section{Funding}

The present study was supported by the Department of Defense through an Exploration-Hypothesis Development Award (grant no. W81XWH-14-1-0255).

\section{Availability of data and materials}

The datasets used and/or analyzed during the present study are available from the corresponding author on reasonable request.

\section{Authors' contributions}

PKV conceived and designed the review, researched the literature and wrote the manuscript.

\section{Ethics approval and consent to participate}

Not applicable.

\section{Patient consent for publication}

Not applicable.

\section{Competing interests}

The author declares that they have no competing interests.

\section{References}

1. American Cancer Society: Cancer Facts \& Figures 2015. American Cancer Society, Atlanta, GA, 2015.

2. Baetke SC, Adriaens ME, Seigneuric R, Evelo CT and Eijssen LM: Molecular pathways involved in prostate carcinogenesis: Insights from public microarray datasets. PLoS One 7: e 49831, 2012.

3. Center MM, Jemal A, Lortet-Tieulent J, Ward E, Ferlay J, Brawley $\mathrm{O}$ and Bray F: International variation in prostate cancer incidence and mortality rates. Eur Urol 61: 1079-1092, 2012.

4. Kornmann B: Quality control in mitochondria: Use it, break it, fix it, trash it. F1000Prime Rep 6: 15, 2014.

5. van der Bliek AM, Sedensky MM and Morgan PG: Cell biology of the mitochondrion. Genetics 207: 843-871, 2017.

6. Parr RL, Mills J, Harbottle A, Creed JM, Crewdson G, Reguly B and Guimont FS: Mitochondria, prostate cancer, and biopsy sampling error. Discov Med 15: 213-220, 2013.

7. Boland ML, Chourasia AH and Macleod KF: Mitochondrial dysfunction in cancer. Front Oncol 3: 292, 2013.

8. Costello LC and Franklin RB: The clinical relevance of the metabolism of prostate cancer; zinc and tumor suppression: Connecting the dots. Mol Cancer 5: 17, 2006.

9. Costello LC, Guan Z, Kukoyi B, Feng P and Franklin RB: Terminal oxidation and the effects of zinc in prostate versus liver mitochondria. Mitochondrion 4: 331-338, 2004. 
10. Costello LC and Franklin RB: The intermediary metabolism of the prostate: A key to understanding the pathogenesis and progression of prostate malignancy. Oncology 59: 269-282, 2000.

11. Vayalil PK and Landar A: Mitochondrial oncobioenergetic index: A potential biomarker to predict progression from indolent to aggressive prostate cancer. Oncotarget 6: 43065-43080, 2015.

12. Panov A and Orynbayeva Z: Bioenergetic and antiapoptotic properties of mitochondria from cultured human prostate cancer cell lines PC-3, DU145 and LNCaP. PLoS One 8: e72078, 2013.

13. Andersen KF, Divilov V, Sevak K, Koziorowski J, Lewis JS and Pillarsetty N: Influence of free fatty acids on glucose uptake in prostate cancer cells. Nucl Med Biol 41: 254-258, 2014.

14. Hofer C, Laubenbacher C, Block T, Breul J, Hartung R and Schwaiger M: Fluorine-18-fluorodeoxyglucose positron emission tomography is useless for the detection of local recurrence after radical prostatectomy. Eur Urol 36: 31-35, 1999.

15. Liu Y, Zuckier LS and Ghesani NV: Dominant uptake of fatty acid over glucose by prostate cells: A potential new diagnostic and therapeutic approach. Anticancer Res 30: 369-374, 2010.

16. Diers AR, Vayalil PK, Oliva CR, Griguer CE, Darley-Usmar V, Hurst DR, Welch DR and Landar A: Mitochondrial bioenergetics of metastatic breast cancer cells in response to dynamic changes in oxygen tension: Effects of HIF-1 $\alpha$. PLoS One 8: e68348, 2013.

17. Kramer PA, Chacko BK, Ravi S, Johnson MS, Mitchell T, Barnes S, Arabshahi A, Dell'Italia LJ, George DJ, Steele C, et al: Hemoglobin-associated oxidative stress in the pericardial compartment of postoperative cardiac surgery patients. Lab Invest 95: 132-141, 2015.

18. Franklin RB, Zou J, Yu Z and Costello LC: EAAC1 is expressed in rat and human prostate epithelial cells; functions as a high-affinity L-aspartate transporter; and is regulated by prolactin and testosterone. BMC Biochem 7: 10, 2006.

19. Mayers JR and Vander Heiden MG: Nature and nurture: What determines tumor metabolic phenotypes? Cancer Res 77: 3131-3134, 2017

20. Parr RL, Dakubo GD, Thayer RE, McKenney $\mathrm{K}$ and Birch-Machin MA: Mitochondrial DNA as a potential tool for early cancer detection. Hum Genomics 2: 252-257, 2006.

21. Vayalil PK, Oh JY, Zhou F, Diers AR, Smith MR, Golzarian H, Oliver PG, Smith RA, Murphy MP, Velu SE and Landar A: A novel class of mitochondria-targeted soft electrophiles modifies mitochondrial proteins and inhibits mitochondrial metabolism in breast cancer cells through redox mechanisms. PLoS One 10: e0120460, 2015

22. Smith MR, Vayalil PK, Zhou F, Benavides GA, Beggs RR, Golzarian H, Nijampatnam B, Oliver PG, Smith RA, Murphy MP, et al: Mitochondrial thiol modification by a targeted electrophile inhibits metabolism in breast adenocarcinoma cells by inhibiting enzyme activity and protein levels. Redox Biol 8: $136-148,2016$

23. Wu M, Neilson A, Swift AL, Moran R, Tamagnine J, Parslow D, Armistead S, Lemire K, Orrell J, Teich J, et al: Multiparameter metabolic analysis reveals a close link between attenuated mitochondrial bioenergetic function and enhanced glycolysis dependency in human tumor cells. Am J Physiol Cell Physiol 292: C125-C136, 2007.

24. Schöpf B, Schäfer G, Weber A, Talasz H, Eder IE, Klocker H and Gnaiger E: Oxidative phosphorylation and mitochondrial function differ between human prostate tissue and cultured cells FEBS J 283: 2181-2196, 2016.

25. Chacko BK, Kramer PA, Ravi S, Benavides GA, Mitchell T, Dranka BP, Ferrick D, Singal AK, Ballinger SW, Bailey SM, et al: The bioenergetic health index: A new concept in mitochondrial translational research. Clin Sci (Lond) 127: 367-373, 2014.

26. Brand MD and Nicholls DG: Assessing mitochondrial dysfunction in cells. Biochem J 435: 297-312, 2011.

27. Jastroch M, Divakaruni AS, Mookerjee S, Treberg JR and Brand MD: Mitochondrial proton and electron leaks. Essays Biochem 47: 53-67, 2010.

28. Hill BG, Benavides GA, Lancaster JR Jr, Ballinger S, Dell'Italia L, Jianhua $\mathrm{Z}$ and Darley-Usmar VM: Integration of cellular bioenergetics with mitochondrial quality control and autophagy. Biol Chem 393: 1485-1512, 2012.

29. Dranka BP, Hill BG and Darley-Usmar VM: Mitochondrial reserve capacity in endothelial cells: The impact of nitric oxide and reactive oxygen species. Free Radic Biol Med 48: 905-914, 2010.

30. Hill BG, Dranka BP, Zou L, Chatham JC and Darley-Usmar VM: Importance of the bioenergetic reserve capacity in response to cardiomyocyte stress induced by 4-hydroxynonenal. Biochem J 424: 99-107, 2009
31. Nicolson GL: Mitochondrial dysfunction and chronic disease: Treatment with natural supplements. Integr Med (Encinitas) 13 35-43, 2014

32. Fiaschi T, Marini A, Giannoni E, Taddei ML, Gandellini P, De Donatis A, Lanciotti M, Serni S, Cirri P and Chiarugi P: Reciprocal metabolic reprogramming through lactate shuttle coordinately influences tumor-stroma interplay. Cancer Res 72: 5130-5140, 2012.

33. Shtivelman E, Beer TM and Evans CP: Molecular pathways and targets in prostate cancer. Oncotarget 5: 7217-7259, 2014.

34. Li C, Li Y, He L, Agarwal AR, Zeng N, Cadenas E and Stiles B $\mathrm{PI} 3 \mathrm{~K} / \mathrm{AKT}$ signaling regulates bioenergetics in immortalized hepatocytes. Free Radic Biol Med 60: 29-40, 2013.

35. Lightowlers RN, Chinnery PF, Turnbull DM and Howell N: Mammalian mitochondrial genetics: Heredity, heteroplasmy and disease. Trends Genet 13: 450-455, 1997.

36. Wallace DC and Chalkia D: Mitochondrial DNA genetics and the heteroplasmy conundrum in evolution and disease. Cold Spring Harb Perspect Biol 5: a021220, 2013.

37. Jerónimo $C$, Nomoto $S$, Caballero OL, Usadel H, Henrique R, Varzim G, Oliveira J, Lopes C, Fliss MS and Sidransky D: Mitochondrial mutations in early stage prostate cancer and bodily fluids. Oncogene 20: 5195-5198, 2001.

38. Jessie BC, Sun CQ, Irons HR, Marshall FF, Wallace DC and Petros JA: Accumulation of mitochondrial DNA deletions in the malignant prostate of patients of different ages. Exp Gerontol 37: 169-174, 2001.

39. Chen JZ, Gokden N, Greene GF, Mukunyadzi P and Kadlubar FF: Extensive somatic mitochondrial mutations in primary prostate cancer using laser capture microdissection. Cancer Res 62: 6470-6474, 2002.

40. Chen JZ, Gokden N, Greene GF, Green B and Kadlubar FF: Simultaneous generation of multiple mitochondrial DNA mutations in human prostate tumors suggests mitochondrial hyper-mutagenesis. Carcinogenesis 24: 1481-1487, 2003.

41. Chen JZ and Kadlubar FF: Mitochondrial mutagenesis and oxidative stress in human prostate cancer. J Environ Sci Health C Environ Carcinog Ecotoxicol Rev 22: 1-12, 2004.

42. Petros JA, Baumann AK, Ruiz-Pesini E, Amin MB, Sun CQ, Hall J, Lim S, Issa MM, Flanders WD, Hosseini SH, et al: mtDNA mutations increase tumorigenicity in prostate cancer. Proc Natl Acad Sci USA 102: 719-724, 2005.

43. Dakubo GD, Parr RL, Costello LC, Franklin RB and Thayer RE: Altered metabolism and mitochondrial genome in prostate cancer. J Clin Pathol 59: 10-16, 2006.

44. Gómez-Zaera M, Abril J, González L, Aguiló F, Condom E, Nadal $\mathrm{M}$ and Nunes V: Identification of somatic and germline mitochondrial DNA sequence variants in prostate cancer patients. Mutat Res 595: 42-51, 2006.

45. Higuchi M, Kudo T, Suzuki S, Evans TT, Sasaki R, Wada Y, Shirakawa T, Sawyer JR and Gotoh A: Mitochondrial DNA determines androgen dependence in prostate cancer cell lines. Oncogene 25: 1437-1445, 2006.

46. Parr RL, Dakubo GD, Crandall KA, Maki J, Reguly B, Aguirre A, Wittock R, Robinson K, Alexander JS, Birch-Machin MA, et al: Somatic mitochondrial DNA mutations in prostate cancer and normal appearing adjacent glands in comparison to age-matched prostate samples without malignant histology. J Mol Diagn 8: 312-319, 2006

47. Khandrika L, Kumar B, Koul S, Maroni P and Koul HK: Oxidative stress in prostate cancer. Cancer Lett 282: 125-136, 2009.

48. Kloss-Brandstätter A, Schäfer G, Erhart G, Hüttenhofer A, Coassin S, Seifarth C, Summerer M, Bektic J, Klocker H and Kronenberg F: Somatic mutations throughout the entire mitochondrial genome are associated with elevated PSA levels in prostate cancer patients. Am J Hum Genet 87: 802-812, 2010

49. Ashtiani ZO, Heidari M, Hasheminasab SM, Ayati M and Rakhshani N: Mitochondrial D-Loop polymorphism and microsatellite instability in prostate cancer and benign hyperplasia patients. Asian Pac J Cancer Prev 13: 3863-3868, 2012.

50. Arnold RS, Sun Q, Sun CQ, Richards JC, O'Hearn S, Osunkoya AO, Wallace DC and Petros JA: An inherited heteroplasmic mutation in mitochondrial gene COI in a patient with prostate cancer alters reactive oxygen, reactive nitrogen and proliferation. Biomed Res Int 2013: 239257, 2013.

51. Arnold RS, Fedewa SA, Goodman M, Osunkoya AO, Kissick HT, Morrissey C, True LD and Petros JA: Bone metastasis in prostate cancer: Recurring mitochondrial DNA mutation reveals selective pressure exerted by the bone microenvironment. Bone 78: 81-86, 2015. 
52. Dong YL, Luan ZM, Xue ZY and Li YJ: Complete mitochondrial genome sequence and mutations of the prostate cancer model inbred Sprague-Dawley strain. Mitochondrial DNA A DNA Mapp Seq Anal 27: 2266-2267, 2016.

53. Milosevic M, Warde P, Ménard C, Chung P, Toi A, Ishkanian A, McLean M, Pintilie M, Sykes J, Gospodarowicz M, et al: Tumor hypoxia predicts biochemical failure following radiotherapy for clinically localized prostate cancer. Clin Cancer Res 18 $2108-2114,2012$

54. Solaini G, Baracca A, Lenaz G and Sgarbi G: Hypoxia and mitochondrial oxidative metabolism. Biochim Biophys Acta 1797: 1171-1177, 2010.

55. Feeley KP, Westbrook DG, Bray AW and Ballinger SW: An ex-vivo model for evaluating bioenergetics in aortic rings. Redox Biol 2: 1003-1007, 2014.

56. Schuh RA, Clerc P, Hwang H, Mehrabian Z, Bittman K, Chen H and Polster BM: Adaptation of microplate-based respirometry for hippocampal slices and analysis of respiratory capacity. J Neurosci Res 89: 1979-1988, 2011.

57. Perry CG, Kane DA, Lanza IR and Neufer PD: Methods for assessing mitochondrial function in diabetes. Diabetes 62: 1041-1053, 2013

58. de Graaf IA, Olinga P, de Jager MH, Merema MT, de Kanter R, van de Kerkhof EG and Groothuis GM: Preparation and incubation of precision-cut liver and intestinal slices for application in drug metabolism and toxicity studies. Nat Protoc 5: 1540-1551, 2010.
59. Martin C, Uhlig S and Ullrich V: Videomicroscopy of methacholine-induced contraction of individual airways in precision-cut lung slices. Eur Respir J 9: 2479-2487, 1996.

60. Poosti F, Pham BT, Oosterhuis D, Poelstra K, van Goor H, Olinga P and Hillebrands JL: Precision-cut kidney slices (PCKS) to study development of renal fibrosis and efficacy of drug targeting ex vivo. Dis Model Mech 8: 1227-1236, 2015.

61. Lanza IR and Nair KS: Mitochondrial metabolic function assessed in vivo and in vitro. Curr Opin Clin Nutr Metab Care 13: 511-517, 2010.

62. Marcinek DJ: Mitochondrial dysfunction measured in vivo. Acta Physiol Scand 182: 343-352, 2004

63. Amara CE, Marcinek DJ, Shankland EG, Schenkman KA, Arakaki LS and Conley KE: Mitochondrial function in vivo: Spectroscopy provides window on cellular energetics. Methods 46: 312-318, 2008.

64. Campbell MD and Marcinek DJ: Evaluation of in vivo mitochondrial bioenergetics in skeletal muscle using NMR and optical methods. Biochim Biophys Acta 1862: 716-724, 2016.

This work is licensed under a Creative Commons Attribution-NonCommercial-NoDerivatives 4.0 International (CC BY-NC-ND 4.0) License. 